\title{
La provisión de servicios sanitarios en el ámbito público: una revisión crítica en torno al concepto de cuasimercado
}

\author{
Aleix Gregori \\ Universitat Rovira i Virgili \\ aleix.gregori@urv.cat
}

Resumen: En este artículo se realiza una revisión de la literatura concerniente al concepto de cuasimercado, también llamado mercado interno. Este concepto ha servido de fundamento teórico de las politicas recientes de prestación de servicios sanitarios en los sistemas de atención universal, en particular dando cobertura a la entrada de proveedores privados en la sanidad pública. A partir de un análisis crítico de esa literatura, se pone de manifiesto que dicho concepto es confuso y está basado en una serie de juicios a priori relativos a la idea neoclásica de competencia perfecta. Por ello, la definición de cuasimercado no constituye una base adecuada para el análisis de las prácticas que se ban llevado a cabo en los últimos años en el ámbito de la sanidad pública universal. El reconocimiento de las limitaciones que entraña el concepto de cuasimercado permite clarificar algunos elementos de análisis y puede guiar futuras lineas de investigación en esta materia.

Palabras clave: cuasimercado, mercado interno, estado de bienestar, servicios de salud, política sanitaria.

The provision of public health services: a critical review of the quasi-market concept

Abstract: This article is a review of the literature related to the concept of the quasimarket, also referred to as the internal market. This concept has provided a theoretical foundation for recent provision policies in universal health care systems. In particular, it provides a rationale to support the entry of private providers into the public health 
care network. A critical analysis of the literature demonstrates that the concept is confusing and is based on a series of a priori judgments concerning the neoclassical idea of perfect competition. Hence, the quasi-market definition does not offer an adequate starting point from which to analyze the policies that bave been implemented in universal bealth care in recent years. Recognizing the limitations of the quasimarket concept allows certain analytical elements to be clarified and can guide future research in this area.

Keywords: quasi-market, internal market, welfare state, bealth services, bealth policy. 


\section{Introducción}

La prestación de servicios sanitarios en los países de la Europa Occidental se caracteriza por la voluntad de los estados de garantizar a sus ciudadanos el acceso a una atención sanitaria que les permita mantener unos niveles adecuados de salud. El mecanismo con que se lleva a la práctica dicha voluntad es doble. Por un lado, en países como Francia o Alemania, existe un seguro sanitario obligatorio, cuyas primas se deducen del salario percibido por los trabajadores; en este caso existe, además, una mezcla de provisión pública y privada de servicios sanitarios. Por otro lado, en países como el Reino Unido o España, esa voluntad se concreta en un sistema sanitario con vocación universal, cuya financiación corre a cargo del sistema impositivo general, y cuya provisión se lleva a cabo fundamentalmente a través de proveedores públicos.

Más allá de su vocación universal, sin embargo, en el segundo caso, se ha ido abandonando progresivamente la provisión integrada de los servicios sanitarios a través de un único ente público, y se ha dado paso a fórmulas de gestión de la provisión más flexibles. Ello se debe a la introducción en los mecanismos de prestación del principio de separación de funciones de financiación, compra y provisión de servicios. Este principio propone que el sector público deje de ser quien simultáneamente financie los servicios sanitarios y provea de estos servicios a la ciudadanía, y pase a ser fundamentalmente el administrador de los recursos que financian el sistema. Para ello, una agencia pública se encarga de la función de compra de servicios, mientras que la naturaleza de los centros proveedores es diversa, permitiendo la entrada de proveedores privados en el sistema sanitario público (figura 1). La introducción de estos mecanismos de provisión se remonta a principios de la década de 1990, y se han venido aplicando ampliamente en los mencionados sistemas sanitarios de atención universal.

Ahora bien, el reciente proceso de adjudicación de la gestión de seis hospitales públicos a empresas privadas en la Comunidad de Madrid, así como procesos de concesión similares ocurridos en Cataluña para centros de atención primaria y socio-sanitaria, han puesto nuevamente en el ojo del huracán del debate público la conveniencia de la presencia de proveedores privados en el ámbito de la sanidad pública. Así, en el actual contexto de crisis económica, dichas iniciativas, junto con los recortes presupuestarios en sanidad, han encontrado la oposición de una parte importante de los ciudadanos, los cuales entienden que todo ello constituye un intento de desmantelar el estado de bienestar. En contra de esta visión, se argumenta que aquello que se debe replantear es el modelo de prestación de servicios sanitarios en su conjunto, esgrimiendo el viejo leitmotiv de la necesidad de mejora de la eficiencia en el sector público. 
Figura 1: Esquema básico de provisión sanitaria

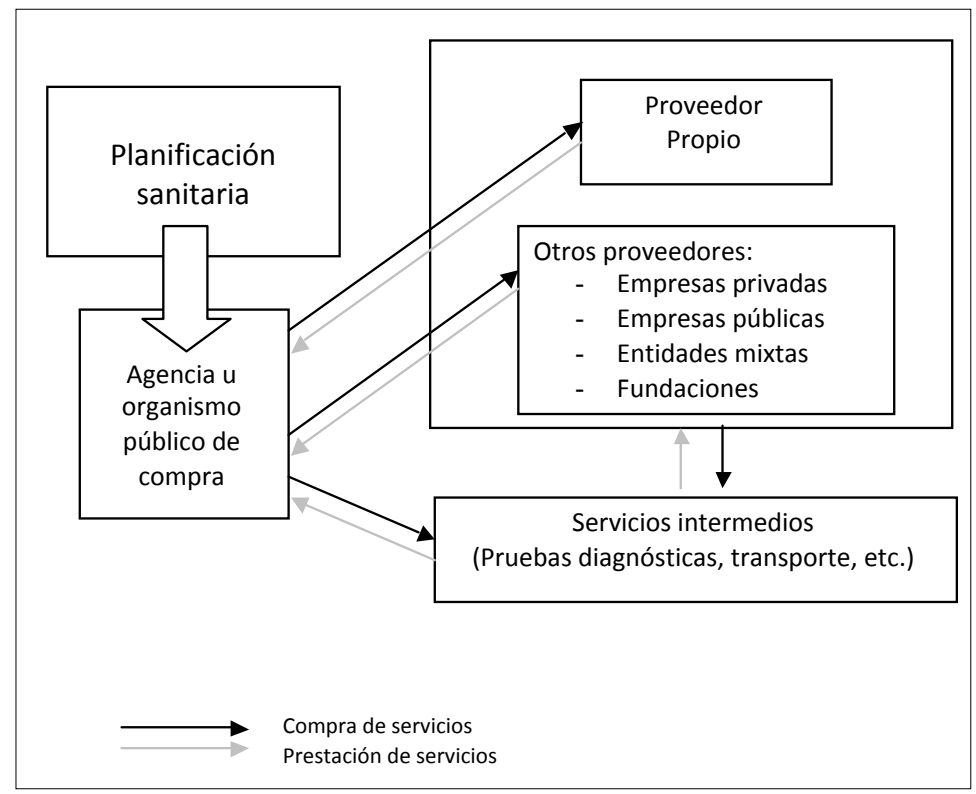

A propósito de este resurgido debate, en el presente artículo se revisa la literatura en torno al concepto de cuasimercado, literatura que representa el fundamento teórico de las prácticas de gestión privada dentro del ámbito público y, a su vez, las legitima. Esta literatura constituye un marco de referencia amplio de los sistemas sanitarios del tipo Servicio Nacional de Salud, tal como son definidos en la actualidad. A un nivel más específico, la llamada narrativa de los cuasimercados aborda los cambios ocurridos en el mecanismo de prestación de servicios sociales desde el ámbito de conocimiento de la economía, partiendo de un análisis normativo del fenómeno. Tanto en esta narrativa como en sus antecedentes, el debate académico gira en torno al concepto de mercado y a la distinción entre este y otros mecanismos de asignación de recursos.

La revisión que planteamos en este trabajo es relevante porque puede ayudar a clarificar algunos conceptos e ideas que subyacen a las políticas sanitarias actuales. Desde una perspectiva académica, esta clarificación va a servir para marcar cuáles son las futuras líneas de investigación que pueden seguir los investigadores sociales interesados en esta materia. El resto de los contenidos del artículo se estructuran de la siguiente manera. En el apartado 2 se hace una descripción sucinta de las tendencias de gestión pública que dieron lugar a la literatura vinculada al concepto de cuasimercado. En el apartado 3 se indaga en el origen de este y otros términos relacionados. En el apartado 4 revisamos el propio concepto de 
cuasimercado a partir de la literatura en que este fue desarrollado. En el apartado 5 realizamos un análisis crítico de dicha literatura con el fin de establecer un nuevo prisma de análisis del papel de los agentes privados en la sanidad pública. Finalmente, en el apartado 6 exponemos algunas conclusiones.

\section{Cambios en los mecanismos de actuación pública: el new public management}

En las últimas décadas se ha producido en la administración pública una serie de reformas encaminadas a sustituir el modelo clásico-burocrático por unas nuevas formas de gestión pública caracterizadas por una mayor flexibilidad. La expresión new public management (NPM) - o nueva gestión pública - se utiliza corrientemente para designar este conjunto de reformas. En general, los objetivos que persigue el NPM son mejorar la eficiencia y la eficacia de la administración pública así como contener sus costes.

En un sentido más amplio, el término NPM hace referencia a toda una doctrina que acompaña a las nuevas prácticas en el seno del sector público; un corpus de ideas que ha conducido a un cambio de paradigma en la administración pública (Batley y Larbi, 2004; Christensen y Lægreid, 2007; Dent et alii, 2004; Dibben et alii, 2004; OECD, 2005). Como tal, bebe de distintas fuentes intelectuales, especialmente procedentes de la literatura económica. El origen del NPM se puede interpretar como la confluencia de dos corrientes distintas de ideas (Hood, 1991): por un lado, la «nueva economía institucional», construida en torno al desarrollo de la teoría de la elección pública, la teoría de los costes de transacción y la teoría de la agencia, y, por otra parte, las sucesivas oleadas de managerialism de corte empresarial en el sector público, en la tradición del movimiento científico internacional de gestión empresarial. Estas dos vertientes han dotado al NPM de contenido doctrinal, generando una serie de principios de reforma basados en ideas como la concurrencia, la elección por parte del usuario, la transparencia y la preocupación por los incentivos, en relación con la nueva economía institucional, y también en la gestión profesional, la discrecionalidad y la mejora de los resultados organizativos, por influencia del managerialism. Las reformas enmarcadas en el NPM constituyen una tendencia generalizada y se han llevado a cabo en varios países de Europa y de la ODCE, con el Reino Unido como caso paradigmático.

Uno de los elementos centrales de esta nueva tendencia es la separación entre los papeles funcionales de comprador y provisor en el sector público y la consiguiente introducción de contratos (Gallego, 2000). Los Estados modernos son 
responsables en la provisión de determinados bienes y servicios que se consideran socialmente deseables como la sanidad o la educación. Son los llamados «bienes preferentes». En un modelo burocrático de gestión pública el Estado provee de un determinado bien preferente a la sociedad, a la vez que lo financia, de manera que las funciones de compra y provisión están integradas en un solo ente público. En el contexto del NPM, muchos Estados han optado por segregar ambos roles. La idea es que las decisiones sobre la compra de los bienes y servicios necesarios para implementar el bien preferente en la sociedad y las decisiones sobre la provisión de estos bienes y servicios no recaigan en la misma entidad jurídica. En cualquier caso es el sector público quien se encarga de la adquisición de los bienes y servicios implicados, como responsable ante la ciudadanía de la provisión de un determinado bien preferente. La provisión efectiva, en cambio, puede ser realizada tanto por una entidad privada, claramente separada de la acción pública, como por una entidad pública distinta del ente público encargado de la función de compra. Esta estructura de relaciones es denominada por diversos autores, desde una perspectiva de análisis económico, como cuasimercado o mercado interno (Barlett, 1991; Le Grand, 1991; Maynard, 1991; Barlett y Le Grand, 1993; Propper, 1993; Chalkley y Malcomson, 1996a, 1996b; Jenkinson y Mayer, 1996; Jones y Cullis, 1996; Deakin y Michie, 1997; Kitchener, 1998; Le Grand et alii, 1998).

Ambos términos, sin embargo, provienen de otras fuentes de la literatura: la teoría de los costes de transacción y la teoría de la empresa. Si bien en su contexto originario, cuasimercado y mercado interno responden a conceptos un tanto diferentes, en el ámbito de las reformas de la gestión pública se utilizan a menudo indistintamente. Mayoritariamente, sin embargo, y a raíz de los trabajos de Le Grand (1991) y Barlett y Le Grand (1993), se impuso, en una línea académica de corte económico, la primera denominación. En este sentido, hablamos de la narrativa de los cuasimercados (McMaster, 2002) para referirnos a la literatura económica que analiza las reformas ocurridas en el estado del bienestar, concretadas en la separación de funciones de compra y provisión por parte de la administración. Antes de entrar en detalle en esta narrativa, vale la pena echar un vistazo a la noción existente de estos términos en la literatura donde se han desarrollado y al debate conceptual y analítico que ha generado. 


\section{En el origen de los términos: cuasimercado y mercado interno}

El primer autor que utilizó la palabra cuasimercado fue Williamson (1975), en el contexto de la economía de los costes de transacción. Aunque sin una definición explícita, el término hace referencia a una institución intermedia entre organización empresarial (jerarquía) y transacción de mercado, descrita como «forma híbrida» (Williamson, 1985). También Ménard (1995) utiliza el término cuasimercado como una desviación de los «conceptos puros»o «categorías puras» de mercado y jerarquía. Otros términos utilizados de forma similar son los de empresa bíbrida (Ménard, 1996) y cuasiempresa (Eccles, 1981). Una idea ligeramente diferente de las anteriores, aunque relacionada, es la de mercado interno dentro de la empresa (Doering y Piore, 1971). También en esta área, y teniendo en cuenta los diferentes matices en cada caso, es posible una cierta confusión terminológica. Así, se pueden encontrar expresiones como organización internamente estructurada como cuasimercado, en referencia a la idea de «mercado interno».

Toda esta nomenclatura que acabamos de revisar es una manifestación de la ambigüedad y la falta de consenso que existe por parte de los economistas en relación con elementos tan importantes como el concepto de empresa, el concepto de mercado y, más allá, los conceptos de organización e institución (Ménard, 1995; Hodgson, 2002). Esta confusión o falta de unicidad conceptual no permite determinar con claridad qué distingue a una empresa de un mercado, lo que conduce a no poder establecer una frontera analítica entre ambas instituciones económicas. Es en este contexto de indeterminación en el que surgen términos usuales como forma híbrida, cuasimercado o mercado interno, fruto de la observación de ciertas estructuras empresariales, en el seno de la organización, similares al mecanismo de mercado.

En este sentido, existen divisiones o departamentos de una misma empresa que realizan transacciones entre sí utilizando mecanismos de facturación o encaje de oferta y demanda que, por analogía, recuerdan el funcionamiento de un mercado de bienes, servicios o factores. Es lo que en la literatura se ha venido a llamar mercado interno o mercado dentro de la empresa. En casos extremos, aquellas divisiones compiten con empresas ajenas para proveer de una determinada mercancía o servicio a su propia organización. Como ejemplos clásicos podemos citar los internal labour markets (Doeringer y Piore, 1971), en relación con la renegociación de contratos de trabajo o a la promoción interna dentro de la empresa, o los internal capital markets (Williamson, 1975) para el presupuesto dividido $y$ asignado por la oficina central de una empresa en el contexto de estructuras 
multidivisionales. Ciertas formas de transferencia que utilizan indicadores de precios o ciertas formas de competencia por unos recursos escasos dentro de la organización son, en general, las características que han llamado la atención de los académicos a la hora de tratar estos fenómenos.

Por otra parte, se habla también de algunas concepciones «híbridas», como cuasiempresas o cuasimercados. Estas ideas hacen referencia fundamentalmente a relaciones verticales entre empresas independientes, generalmente de carácter estable y continuado. Los autores que defienden esa terminología argumentan que sirve para designar un tipo de relaciones que se sitúa en un punto indeterminado entre empresa y mercado, en lo que podríamos calificar como un continuo entre ambos conceptos. Así, Eccles (1981) habla de casi integración para referirse a lo que él llama cuasiempresa. Igualmente, Cheung (1983) se cuestiona si estas empresas no forman una sola, refiriéndose a una integración vertical a través de contratos. Otro ejemplo de estas formas híbridas sería la relación a través de franquicia (Williamson, 1985; Ménard, 1995).

Una explicación a la diversidad de términos empleados para denominar formas organizativas similares y a la ambigüedad analítica en relación con la distinción entre mercado y empresa podría residir en la ausencia de una definición unívoca de esta; Machlup (1967) identifica un mínimo de diez conceptos diferentes empleados para referirse a la empresa en la literatura económica y empresarial. Desde la «caja negra» neoclásica, pasando por la economía de los costes de transacción y los derechos de propiedad, hasta la moderna teoría de la agencia, las diferentes aproximaciones a la teoría de la empresa han estimulado, más que aliviado, la confusión creada en torno a la delimitación de una frontera entre mercado y empresa.

En este sentido, Hodgson (2002) plantea que el abandono progresivo de la concepción legal de la empresa, entendida como entidad con personalidad jurídica, ha conducido directamente a esta problemática y habla del «mito del mercado interno», así como del «mito del híbrido empresa-mercado». El autor argumenta que, de hecho, tales concepciones no pueden justificarse si se tiene en cuenta el elemento legal en la definición de empresa. Así, en relación con la idea de «mercado interno», afirma que «las transferencias internas dentro de la empresa no conllevan el intercambio de derechos legales de propiedad. Los objetos de intercambio' siguen siendo propiedad de la empresa. Estos intercambios no son contratos comerciales que se puedan hacer cumplir legalmente: son trasferencias internas» (Hodgson, 2002: 46). En relación con lo que se ha venido a llamar formas hibridas, concluye que se trata de «contratos relacionales» entre empresas; una tercera opción, alternativa al mercado y la empresa única, más que un elemento situado 
entre una y otra. Con esta perspectiva, mercados y empresas aparecen como conceptos netamente diferenciados: no hay mercados dentro de las empresas ni transacciones contractuales que constituyan una empresa. Contrariamente a las tesis de Hodgson, sin embargo, otros trabajos hablan de la empresa como una «ficción legal» (Alchian y Demsetz, 1972; Jensen y Meckling, 1976; Fama, 1980). Estos autores entienden la empresa como un mero nexo de contratos que se sirve de la consideración legal de la organización como individuo. Inevitablemente, la separación entre mercado y empresa vuelve a quedar difuminada, ya que, en este caso «tiene poco o ningún sentido intentar distinguir aquello que está 'dentro' de la empresa (o cualquier otra organización) de aquello que está 'fuera' de ella. Hay [... ] solamente una multitud de relaciones complejas (esto es, contratos) entre la ficción legal (la empresa) y los propietarios del factor trabajo, los materiales y el capital, así como de la producción» (Jensen y Meckling, 1976: 311). En una aproximación similar, Elling (2001) no considera relevante distinguir entre empresa y mercado sino que defiende que, dada la realidad empresarial, la verdadera distinción se debe hacer entre los mercados externos (a la empresa) y los mercados internos. En este sentido, Elling entiende la empresa (o mercado interno) como un membership club cuyos miembros gozan de acceso a los «bienes públicos» de la empresa, que son aquellos relacionados con el conocimiento, tales como las capacidades generadoras de valor.

Podemos comprobar que la cuestión genera controversia entre los académicos, en posiciones bastante alejadas unas de otras. Sin embargo, la nomenclatura derivada de la literatura que designa las formas híbridas y los mercados internos se ha consolidado con fuerza entre los investigadores de las ciencias sociales y ha sido adoptada en diferentes ámbitos, entre ellos el de la gestión pública.

\section{Reformas en el estado de bienestar: la narrativa de los cuasimercados}

El origen y contextualización del término cuasimercado poco tiene que ver, en principio, con la orientación social del modelo del estado de bienestar y los mecanismos de funcionamiento del sector público. Sin embargo, el advenimiento en Gran Bretaña de una ola de reformas en la provisión pública de servicios dentro del estado de bienestar modificó el destino del término acuñado por Williamson, y se creó una concepción paralela a la desarrollada en su marco de referencia original. A finales de la década de 1980, el entonces gobierno conservador británico inició un programa de cambios «orientados al mercado» en la estructura de 
prestación sanitaria, de educación y de acceso a la vivienda. En este contexto, el término cuasimercado fue adoptado para designar el esquema de funcionamiento resultante de aquel proceso, en un intento por parte de algunos autores de dotar de un marco conceptual y analítico al componente económico de las reformas del estado de bienestar que se estaban produciendo.

La principal característica de este componente de las reformas es la separación de funciones de compra y provisión de servicios por parte del sector público. De esta manera, «la intención es que el Estado deje de ser tanto el financiador como el proveedor de los servicios. En vez de eso, debe convertirse fundamentalmente en un financiador, comprando dichos servicios a una variedad de proveedores privados, benéficos y públicos» (Le Grand, 1991:1257). Además, «las reformas han procurado asegurar la concurrencia en el mercado a través de la formalización de relaciones entre compradores y vendedores en forma de contratos que articulan el volumen, el precio y la calidad que caracterizan las transacciones» (Maynard, 1991:1280). Así, la separación de funciones que establecen las reformas incorpora la presencia de contratos entre el comprador público y los diferentes proveedores, tanto los privados como los públicos.

El primer autor que utiliza el término cuasimercado para referirse a este nuevo mecanismo de compra y provisión de bienes preferentes es Le Grand (1991). El autor define el cuasimercado en contraposición a un mercado convencional, poniendo de relieve las siguientes características diferenciales entre uno y otro: $i$ ) hay organizaciones sin ánimo de lucro compitiendo por los contratos públicos, a veces compitiendo incluso con organizaciones lucrativas; ii) el poder de compra del consumidor no se manifiesta en términos monetarios; iii) los consumidores no operan por sí mismos en el mercado sino que están representados en el mercado por una serie de agentes.

Según Le Grand, los cuasimercados son mercados en tanto que sustituyen la provisión monopolística del Estado por una estructura con competidores independientes. Podemos hablar de cuasimercados si, además, responden a las características que acabamos de enumerar. A partir de este punto emplearemos el término cuasimercado en el sentido que acabamos de apuntar, es decir, refiriéndonos a estructuras con separación de funciones de compra y provisión de servicios dentro del estado de bienestar caracterizadas por los tres puntos enumerados.

En la definición de más arriba, sin embargo, no queda muy claro qué se entiende exactamente por mercado convencional. En otros trabajos vinculados a la literatura de los cuasimercados encontramos, al menos, algunas notas que clarifican el concepto, una de ellas bastante reveladora: el término cuasimercado «se utiliza para significar que el mercado sanitario del Reino Unido difiere en 
gran medida de la noción que tienen los economistas de competencia perfecta» (Kitchner, 1998: 74). Así, entendemos que el concepto de cuasimercado se define tomando como referencia la idea de mercado competitivo. En este sentido, Barlett y Le Grand (1993) plantean una serie de criterios con los que evaluar las reformas llevadas a cabo. Estos criterios incluyen los siguientes puntos: a) eficiencia, entendida como eficiencia productiva, es decir, el menor coste posible para una cantidad o calidad de servicio dada; b) capacidad de respuesta del servicio a las necesidades y preferencias de los individuos; $c$ ) elección de proveedor por parte del usuario, d) equidad, entendida como el acceso al servicio por parte de los individuos que presenten una mayor necesidad de este, independientemente de factores como los ingresos, el estatus socioeconómico, el género o el origen étnico. Todos los puntos que acabamos de mencionar, con la notable excepción del último, son propiedades inherentes al funcionamiento de un mercado de competencia perfecta. De esta manera podemos entender que la concepción que toman estos autores de la idea cuasimercado es la de una aproximación «equitativa» a las «excelencias» del mercado competitivo.

El éxito de las reformas materializadas en los cuasimercados en el sentido de Le Grand (1991) depende en gran medida del cumplimiento, parcial o total, de una serie de condiciones. Estas hacen referencia a cinco aspectos concretos (Barlett y Le Grand, 1993):

1. Estructura de mercado. La provisión de servicios debería llevarse a cabo en condiciones de competencia; es decir, debería haber un número elevado de compradores y un número elevado de proveedores, para que ninguno de ellos tuviera capacidad para modificar el precio de mercado. El precio debería reflejar, pues, la interacción de la oferta y la demanda del servicio, y poder variar libremente en respuesta a cambios en las condiciones tanto de la oferta como de la demanda. La cuestión de la equidad queda, en este caso, al margen.

2. Información. Para una asignación eficiente de servicios, se requiere que ambos lados de un mercado tengan acceso a información precisa y a bajo costo, especialmente en cuanto a la calidad y los costes de los servicios en cuestión. Así, los proveedores deben ser capaces de establecer los costes de sus actividades para fijar el precio adecuadamente; los compradores, por otra parte, deben poder controlar la calidad del servicio que adquieren.

3. Costes de transacción e incertidumbre. Ciertas transacciones de mercado son bastante complejas y multidimensionales. En estos casos existen costes asociados a la redacción, negociación y salvaguarda de acuerdos de 
intercambio (costes ex ante) y costes asociados al control de los resultados del intercambio para comprobar el cumplimiento de los acuerdos (costes ex post). Estos costes de transacción pueden ser especialmente intensos cuando hay incertidumbre sobre las consecuencias del intercambio y sobre las circunstancias en que se puede producir este intercambio.

4. Motivación. Tanto los proveedores como los compradores de servicios deberían perseguir un fin específico que responda a principios establecidos como necesarios para la relevancia práctica de los cuasimercados que estamos tratando. Por un lado, los proveedores deberían estar motivados como mínimo en parte por consideraciones de carácter financiero. Si no es el caso, es decir, si no persiguen la obtención de beneficios, no responderán como se debe a las señales de mercado y no se alcanzará una situación eficiente. Por otra parte, para dar respuesta adecuadamente a las necesidades y deseos de los usuarios, los compradores deben estar motivados por la persecución del bienestar de estos.

5. Cream-skimming. Esta condición está relacionada explícitamente con la cuestión de la equidad. La expresión cream skimming hace referencia a una situación de discriminación por parte de compradores o proveedores de aquellos usuarios más costosos en términos monetarios. Ejemplos de este perfil serían los enfermos crónicos o los niños problemáticos en la escuela. Si los compradores pueden escoger para quién compran los servicios y los proveedores pueden elegir a quién proveen, entonces los servicios del estado de bienestar podrían no llegar a las personas más necesitadas $y$, por tanto, el objetivo de equidad podría no alcanzarse.

Se puede afirmar que muchas de las condiciones señaladas no acaban de encajar bien en la realidad de los cuasimercados, en particular en los de provisión sanitaria. En primer lugar, la posibilidad de competencia entre proveedores de sanidad está limitada por la presencia usual de proveedores dominantes en el ámbito geográfico de referencia para los usuarios. Tampoco en el lado de la demanda existe, en muchas ocasiones, un número significativo de compradores. La agencia pública encargada de la compra de servicios es, muchas veces, el único agente comprador, si no a nivel nacional o regional, sí a nivel local. Este hecho, sin embargo, puede no ser negativo del todo. Se puede argumentar que, en el caso correspondiente, la existencia de un gran comprador puede mitigar el poder de los proveedores dominantes e incluso convertirse en esencial para contrarrestar sus efectos negativos. De hecho, para los cuasimercados, la ausencia de «competencia» entre compradores no se considera per se una característica negativa. En este sentido, «la planificación es más sencilla con grandes compradores. Solo ellos 
pueden valorar adecuadamente las necesidades de la comunidad y asegurar que todas las instalaciones apropiadas para dicho fin estén provistas. [...] los grandes compradores pueden incurrir en grandes volúmenes de compra y de esta manera disminuir los costes» (Barlett y Le Grand, 1993: 20).

Sin embargo, la cantidad de agentes proveedores o compradores no es el único rasgo que distancia los cuasimercados de las propiedades de la competencia. También el mecanismo de precios actúa de una manera diferente. En los servicios de salud, los precios de los servicios se determinan a partir de un proceso de negociación contractual entre el comprador y proveedores. De este modo, los precios establecidos en este cuasimercado no son el resultado de la interacción «descentralizada» de oferta y demanda, o precios de libre mercado, sino que más bien son lo que podríamos llamar precios administrados o precios negociados. Por otra parte, al tratarse de un mecanismo indirecto, el consumidor final de un cuasimercado o el usuario del servicio no puede influir en el precio final, ya que este es mediado a través de la agencia pública correspondiente. Así, la formación del precio no parece que vaya a reflejar las preferencias de los usuarios, a no ser que la agencia en cuestión sea muy sensible a las necesidades del consumidor final. De la misma manera, en el lado de la oferta, la presencia de una gran variedad de formas de propiedad y control en la gestión de los centros -incluyendo titularidad gubernamental, municipal, y de organizaciones sin ánimo de lucro- puede representar también un problema para la eficiencia. Se puede observar que, dada esta multiplicidad de fórmulas de gestión, la determinación del precio puede no producirse en un contexto de contabilidad de costes, generación de beneficios o restricciones de presupuesto. Es más, si los proveedores en cuestión disponen de cierta capacidad para influir en la determinación del precio, este puede no reflejar adecuadamente una restricción de costes.

Los dos últimos aspectos tratados, la compra indirecta y la presencia de proveedores no restringidos por los costes o no lucrativos, afecta también otro de los puntos comentados: la motivación. Por un lado, la falta de ánimo de lucro o restricción, así como la poca motivación comercial o financiera de muchos de los trabajadores del sector de la provisión sanitaria, podría impedir que los proveedores respondieran a las señales de mercado. Por el otro, cuando un agente actúa como comprador en nombre de los usuarios, existe siempre el problema de asegurar que este realmente actuará teniendo en cuenta las necesidades de los usuarios y no siguiendo sus propios intereses.

El hecho es que, como hemos señalado anteriormente, en los cuasimercados el acceso a los servicios no se determina a través del poder de compra de los individuos, sino mediante un tercer agente que realiza la función de compra. El ob- 
jetivo que se persigue es la equidad distributiva, buscando una correspondencia entre las necesidades individuales y el consumo realizado. Sin embargo, en esta estructura el proveedor sanitario contratado puede tener tendencia a discriminar a los pacientes con mayor riesgo, para reducir sus costes operativos. En este caso hablamos de una situación de cream-skimming que impide alcanzar el objetivo de equidad.

En la prestación de servicios sanitarios es probable que existan también problemas vinculados a la información, tal como en su momento enfatizó Arrow (1963). En particular, pueden surgir comportamientos oportunistas por parte de los proveedores, dado un contexto de asimetría informativa. En este caso el proveedor tiene más información sobre la tecnología y las condiciones en que se presta el servicio. La presencia de muchos provisores (competencia en el lado de la oferta) reduce notablemente la tendencia al oportunismo, ya que el proveedor se enfrenta a la posibilidad de no renovación del contrato si el comportamiento oportunista es detectado. En cambio, si el número de proveedores es reducido, entonces estos pueden extraer renta del comprador o bien realizar un provisión ineficiente, o ambas cosas, incrementando artificialmente el precio que pagar por un nivel determinado de servicio (Propper, 1993; Chalkley y Malcomson, 1996a). Una cuestión relacionada es la calidad del servicio; en este contexto, el proveedor puede disminuir sus costes reduciendo la calidad de la prestación.

La existencia de contratos, sin embargo, limita en otro sentido la introducción de los cuasimercados: la magnitud de los costes de transacción. Si a las características de incertidumbre y racionalidad limitada unimos la asimetría de información que acabamos de apuntar, los costes de redacción y especificación de contratos más completos, que eviten al máximo el comportamiento oportunista, así como los costes administrativos para el control de estos contratos, pueden ser letales para las aspiraciones de un cuasimercado en términos de eficiencia. Esta es una consecuencia directa de la separación de estructuras anteriormente integradas. El modelo tradicional de prestación de servicios sociales por parte del sector público, como la sanidad, es similar a la estructura de grandes empresas verticalmente integradas. Tal como señaló Williamson (1975), en muchas ocasiones la «jerarquía» es más ventajosa en la asignación de recursos que las transacciones realizadas en un contexto de mercado a través de contratos, debido precisamente a la presencia de elevados costes de transacción.

La importancia del elemento contractual para el funcionamiento de estas estructuras ha hecho que la literatura en torno a las cuasimercados se haya evolucionado sobre la base del análisis de las relaciones de agencia (Chalkley y Malcomson, 1998, 2000; Levaggi, 1996, 2005, 2007). Esta literatura analiza el 
elemento de asimetría de información entre los agentes, t trata de determinar los contratos o mecanismos óptimos de pago al proveedor.

\section{Discusión}

El concepto de cuasimercado ha servido de sustento teórico a las nuevas prácticas de gestión sanitaria, y las principales premisas que se derivan de su análisis han guiado las políticas públicas que se han desarrollado en buena parte de los sistemas sanitarios con vocación de atención universal. Aún así, la literatura en torno a los cuasimercados no está exenta de críticas. McMaster $(2001,2002)$ argumenta que esta narrativa no puede dar cuenta de las reformas del estado de bienestar, ya que está concebida como un análisis desvinculado del entorno social y de una aproximación evolucionista necesaria para abordar el cambio institucional que representan esas reformas. Contrariamente, el concepto de cuasimercado se define en relación con el de mercado de competencia perfecta y, por tanto, con una idealización neoclásica de carácter estático en su noción de equilibrio que busca extraer los fenómenos económicos de los procesos históricos y sociales. Más allá, el propio uso del término cuasimercado es impreciso. Dado que se entiende como algo que difiere de un mercado convencional, si este último se define como una estructura de competencia perfecta, entonces otras estructuras, por ejemplo un monopolio, que convencionalmente son entendidas como formas de mercado, podrían llamarse «cuasi» mercados. Lo cierto es que el término cuasimercado usado en relación con las reformas del estado de bienestar ha ido desapareciendo de la literatura en el campo de la economía, y actualmente es más habitual la expresión mercado interno. De todos modos, dicha expresión hace referencia a la misma estructura de relaciones y, en definitiva, al mismo concepto.

Más allá de que las anteriores críticas u otras razones hayan propiciado el desuso de aquella acepción del término, creemos que la definición que da Le Grand (1991) genera confusión por razones adicionales. En primer lugar, al delimitar las características diferenciales respecto del mercado convencional, Le Grand plantea la presencia de entidades o empresas sin ánimo de lucro en el lado de la oferta como una motivación para el uso de la partícula «cuasi». Entendemos que esta es de nuevo una manifestación de una concepción estrecha del intercambio dada por la remisión a la idea de mercados competitivos en el sentido neoclásico; si se entiende el mercado como una institución social de intercambio (Hodgson, 1988) la presencia de empresas no lucrativas no es óbice para la existencia del mercado. En segundo lugar, la definición introduce la idea de que en el lado de la demanda los consumidores no ejercen su poder en la adquisición de los servi- 
cios en términos monetarios. Es decir, el consumidor no paga directamente por el servicio que quiere utilizar. Pero ¿quién se encuentra realmente en el lado de demanda en la estructura que plantea Le Grand? En este caso hay una confusión entre los términos consumidor y usuario. Estrictamente, en el mecanismo de cuasimercado, los ciudadanos no son «compradores» ni clientes de los servicios sanitarios públicos sino que son usuarios. Quien demanda en realidad es el Estado, que debe garantizar la provisión de los servicios en el estado del bienestar. Y el poder de compra en términos monetarios lo ejerce la agencia pública encargada de adquirir los servicios para los usuarios. Creemos, pues, que en este caso no se puede analizar conjuntamente la estructura de compra y provisión de los servicios planteando la presencia de los usuarios o consumidores finales en el lado de la demanda, ya que esto genera dudas sobre quién es realmente demandante en esta estructura.

En este sentido, es la relación entre el Estado, en tanto que comprador de servicios sanitarios, y los proveedores de estos servicios la estructura que debe ser objeto de análisis. Dicha estructura constituye un mercado sin necesidad de ninguna matización adicional. Un mercado posiblemente más regulado o con un mayor nivel de intervención pública que otros, pero un mercado a fin cuentas. Nos adherimos aquí a la postura de Hodgson (2002), en el sentido de que es la naturaleza legal de las transacciones - en tanto que transferencias de derechos de propiedad - lo que delimita un mercado de otras estructuras de asignación de recursos. Asimismo, el hecho de que una transacción se produzca entre dos sujetos legalmente diferenciados es suficiente para que tal intercambio pueda denominarse de mercado. De aquí que el uso de términos o expresiones como mercado interno o cuasimercado, referidas a un mecanismo de prestación de servicios sanitarios con separación de funciones, nos parezca innecesario, confuso e incluso equívoco.

Una vez clarificado esto, cabe plantearse cuál es el beneficio de implementar un mecanismo de separación de funciones en el ámbito sanitario público, y de posibilitar con ello la entrada de proveedores privados. Del análisis de la literatura efectuado en el apartado anterior se desprende que, para algunos autores, la competencia que se genera entre los distintos proveedores, cualquiera que sea su naturaleza, para conseguir contratos con el comprador público es el motor de la obtención de mejoras en términos de capacidad de respuesta, calidad y eficiencia, consideradas en relación con una estructura integrada. Este último aspecto, la eficiencia, no es baladí, ya que remite directamente a la sostenibilidad del sistema público, dada la escasez de recursos, y es uno de los argumentos generalmente esgrimidos para justificar la contratación externa de servicios de salud, y en 
particular la gestión privada estos servicios. En este sentido, hay que hacer dos consideraciones. Por un lado, el grado de competencia entre proveedores sanitarios suele ser escaso. Ello se debe a que, como se ha apuntado anteriormente, el número de estos proveedores en sus respectivas áreas de influencia es reducido, hecho por el cual una estructura de oligopolio es la más usual en mercados localizados geográficamente. Además, la entrada de posibles competidores en estos mercados está altamente regulada, con lo que tampoco existe una elevada presión competitiva por parte de proveedores potenciales. De hecho, los propios autores seminales admiten que en el caso británico la introducción de competencia entre proveedores sanitarios fue, en el mejor de los casos, escasa (Le Grand et alii, 1998). Por otro lado, privado no es sinónimo de eficiente. Uno de los países que más confía en el sector privado para el aseguramiento y la provisión sanitaria es Estados Unidos y, sin embargo, este es uno de los países con mayor nivel de gasto sanitario en relación con su PIB (Mas, 2005).

En suma, parece poco claro que la competencia entre proveedores o el hecho que estos sean privados conlleve un mayor nivel de eficiencia. Algunos trabajos empíricos que han estudiado cambios en los modelos de prestación de servicios sanitarios públicos consistentes con el principio de separación de funciones han observado una mejora de la eficiencia productiva de los proveedores (Tambour y Rehnberg, 1997; Gerdtham et alii 1999a y 1999b). Sin embargo, estos trabajos no demuestran que esta mejora se deba al nivel de competencia, ni tampoco a la presencia de proveedores privados, sino que se limitan a observar un incremento de la eficiencia productiva a lo largo del tiempo. El único trabajo que vincula directamente nivel competitivo y eficiencia en este contexto es el de Dalmau-Matarrodona y Puig-Junoy (1998). Usando datos de corte transversal, estos autores encuentran cierta evidencia a favor de un efecto positivo del grado de competencia sobre la eficiencia de los proveedores, aunque esta evidencia es débil. Por todo ello, el mecanismo por el cual la introducción de la separación de funciones puede mejorar los niveles de eficiencia en la provisión de servicios sanitarios permanece en buena medida inexplicado. En este sentido, otra posible fuente de generación de eficiencia podría residir en el poder de negociación que puede ejercer el comprador público frente a sus proveedores. En un trabajo reciente, Siciliani y Stanciole (2013) ponen énfasis en el uso que una agencia pública encargada de la función de compra puede hacer de ese elemento en su favor.

Más allá de esto, la entrada de proveedores privados en la sanidad pública suscita problemáticas adicionales. En primer lugar, hay que puntualizar que la cesión de la gestión de centros sanitarios de la red pública a entidades privadas no constituye un proceso privatizador stricto sensu, en el sentido de que el Estado 
no se desentiende del servicio que se presta. En cierto modo, la separación de funciones equipara la cobertura sanitaria pública universal a un esquema tradicional de seguro sanitario, aunque con distintos actores. Así, siendo el ciudadano el sujeto asegurado, la autoridad sanitaria ejercería el rol de tomador del seguro, en tanto que financiador de la sanidad pública por mandato del poder legislativo. El organismo designado para la compra de servicios sanitarios ejercería el papel de aseguradora, en tanto que ente responsable del acceso a la sanidad de los ciudadanos, mientras que la provisión efectiva de los servicios correspondería a los proveedores de distinta índole a los cuales ese organismo contrata la atención sanitaria. Este esquema derivado de la separación de funciones se viene aplicando a los sistemas sanitarios de atención universal desde hace más de tres décadas. Buen ejemplo de ello es el modelo de concertación hospitalaria en Cataluña (Pastor, 2006), donde la prestación de servicios es llevada a cabo por entidades gestoras de naturaleza diversa, muchas de ellas ajenas a la administración autonómica - fundamentalmente, centros gestionados por otras administraciones, por consorcios y por entidades privadas sin ánimo de lucro-. Aun así, lo que genera dudas en relación con esta estructura de relaciones es la tendencia actual a dar entrada en el sistema a proveedores gestionados por empresas privadas con ánimo de lucro, tendencia que se inició en España con el llamado modelo Alzira. Y es que el objetivo de generación de beneficios monetarios es juzgado por muchos como incompatible con los valores de un estado de bienestar con vocación universal. Desde la literatura de los cuasimercados se apuntó que las motivaciones lucrativas de los proveedores son necesarias para alcanzar el objetivo de eficiencia. Sin embargo, la lógica tendencia a la minimización de costes por parte de estos proveedores puede tener efectos negativos sobre la calidad del servicio, entendida como la correcta solución de los problemas de salud de los usuarios, y puede generalizar las prácticas de cream-skimming, ya comentadas anteriormente. Ambos efectos son claramente contrarios al espíritu del estado de bienestar universal, cuya justificación reside esencialmente en la idea de merit good o «bien preferente» en términos de preferencias o valores de la comunidad (Musgrave, 1959, 1987). Por otro lado, la concesión de contratos a empresas privadas por parte de la administración conlleva también la posibilidad de conductas corruptas por parte de los agentes que, si cabe, serían todavía más reprobables tratándose de actividades vinculadas al estado de bienestar. Dado que los contactos derivados de estos contratos se repiten en el tiempo, existe, asimismo, la posibilidad de captura del regulador, que pude manifestarse tanto en un trato favorable a dichas empresas por parte de la administración como en un transvase de cargos públicos al sector privado. 
En definitiva, la entrada de proveedores privados con ánimo de lucro en la sanidad pública no está exenta de hándicaps ni es tampoco ajena al debate político y académico. Desde esta perspectiva, sería conveniente explorar las consecuencias que acarree la entrada de estos proveedores sobre elementos tales como la eficiencia en la provisión, la calidad del servicio, la equidad en el acceso o la propia calidad del funcionamiento de las instituciones democráticas.

\section{Conclusiones}

En este artículo se ha revisado la literatura en torno al concepto de cuasimercado, también llamado mercado interno. A partir de esta revisión se han analizado críticamente algunos aspectos relativos a dicho concepto, cuyo interés reside en el actual debate abierto sobre la provisión de servicios sanitarios públicos por parte de empresas privadas. Desde nuestro punto de vista, la idea de cuasimercado es confusa y no permite analizar el fenómeno de la separación entre comprador y proveedor desde un ángulo adecuado. En este sentido, las bondades de esta estructura, tal como se definen en la literatura, están basadas en una serie de apriorismos relativos a la idea neoclásica de competencia perfecta, la cual tiene difícil encaje en los mercados sanitarios.

Por ello, creemos que el abandono del marco conceptual derivado de la literatura de los cuasimercados abre nuevas líneas de investigación que pueden aprovechar la tendencia actual a la gestión privada dentro del ámbito sanitario público universal. Por un lado, es necesario conocer qué mecanismos intrínsecos a la separación de funciones redundan en ganancias de eficacia o eficiencia en la provisión. Por otro lado, sería interesante analizar las consecuencias que puede tener la entrada de empresas con ánimo de lucro en la red pública de prestación de servicios sanitarios dentro del estado de bienestar.

\section{Bibliografía}

Alchian, A. y Demsetz, H. (1972). «Production, information costs and economic organization». American Economic Review, 62 (5): 777-795.

Arrow, K. J. (1963). «Uncertainty and the welfare economics of medical care». American Economic Riview, 53 (5): 941-973.

BARlett, W. (1991). «Quasi-markets and contracts: A markets and hierarchies perspective on NHS reform». Public Money and Management, 11 (3):53-61. 
Barlett, W. y Le Grand, J. (1993). «The theory of quasi-markets», en J. Le Grand, y W. Barlett (Eds.) Quasi-markets and social policy. London: McMillan.

Batley, R. y Larbi, G. A. (2004). The changing role of government: The reform of public services in developing countries. New York: Palgrave Macmillan.

Chalkley, M. y Malcomson, J. M. (1998). «Contracting for health services when patient demand does not reflect quality». Journal of Health Economics, $17(1): 1-19$.

- (1996a). «Competition in NHS quasi-markets». Oxford Review of Economic Policy, 12 (4): 89-99.

- (1996b). «Contracts for the national health service». The Economic Journal, 106 (4): 1691-1701.

- (2000). «Government purchasing of health services», en A. J. Culyer, y J. P. Newhouse (Eds.) Handbook of Health Economics. Amsterdam: NorthHolland.

Cheung, S. N. S. (1983). «The contractual nature of the firm». Journal of Law and Economics, 26 (2): 1-21.

Christensen, T. y Legreid, P. (2007). Transcending new public management: The transformation of public sector reforms. Aldershot: Ashgate.

Deakin, S. y Michie, J. (eds.). (1997). Contracts, co-operation and competition: Studies in economics, management and law. Oxford: Oxford Univertity Press.

Dent, M.; Chandler, J. y Barry, J. (2004). Questioning the new public management. Aldershot: Ashgate.

Dibben, P.; Wood, G. y Roper, I. (2004). Contesting public sector reforms: Critical perspectives, international debates. New York: Palgrave Macmillan.

Doeringer, P. B. y Piore, M. J. (1971). Internal labor markets and manpower analysis. Lexington, MA: Heath.

Eccles, R. G. (1981). «The quasifirm in the construction industry». Journal of Economic Behavior and Organization, 2 (4): 335-357.

Elling, J. (2001). «Internal markets and the theory of the firm». Managerial and Decision Economics, 22 (4-5): 227-237.

FAMA, E. (1980). «Agency theory and the theory of the firm». Journal of Political Economy, 88 (1): 288-307.

Gallego, R. (2000). «Introducing Purchaser/Provider separation in the Catalan health administration: A budget analysis». Public Administration, 78 (2): 423-442. 
Gerdtham, U. G.; Löthgren, M.; Tambour, M. y Rehnberg, C. (1999a). "Internal markets and health care efficiency: A multiple-output stochastic frontier analysis». Health Economics, 8 (2): 151-164.

Gerdtham, U. G*; Rehnberg, C. y Tambour, M. (1999b). «The impact of internal markets on health care efficiency: Evidence from health care reforms in Sweden». Applied Economics, 31 (8): 935-945.

Hodgson, G. M. (1988). Economics and institutions: A manifesto for a modern institutional economics. Cambridge, MA: Polity Press.

Hodgson, G. M. (2002). «The legal nature of the firm and the myth of the firmmarket hybrid». International Journal of Economics and Business, 9 (1):37-60.

Hood, C. (1991). «A public management for all seasons?» Public Administration, $69(1): 3-19$.

Jenkinson, T. y Mayer, C. (1996). "The assessment: Contracts and competition». Oxford Review of Economic Policy, 12 (4):1-10.

Jensen, M. y Meckling, W. H. (1976). «Theory of the firm: Managerial behavior, agency costs and ownership structure». Journal of Financial Economics, 3: 305-360.

Jones, P. R. y Cullis, J. G. (1996). «Decision making in quasi-markets: A pedagogic analysis». Journal of Health Economics, 15: 187-208.

Kitchener, M. (1998). "Quasi-market transformation: An institutionalist approach to change in UK hospitals». Public Administration, 76 (1): 73-95.

Le Grand, J. (1991). «Quasi-markets and social policy». The Economic Journal, 101 (4): 1256-1267.

Le Grand, J.; Mays, N. y Mulligan, J. (1998). Learning from the NHS internal market: A review of the evidence. London: King's Fund.

Levaggi, R. (1996). «NHS contracts: An agency approach». Health Economics, $5(4) \div 341-352$.

Levaggi, R. (2005). «Hospital health care: Pricing and quality control in a spatial model with asymmetry of information». International Journal of Health Care Finance and Economics, 5 (4): 327-349.

- (2007). «Regulating internal markets for hospital care»+ Journal of Regulatory Economics, 32 (2): 173-193.

Machlup, F. (1967). «Theories of the firm* Marginalist, behavioral, managerial». American Economic Review, 57 (1): 1-33.

Mas, N. (2005). «Entorno socio-económico de los sistemas de salud», en J. RIbera; J. A. Gutiérrez \& M. Rosenmöller (eds.) Gestión en el sector de la salud. Madrid: Pearson Educación. 
Maynard, A. (1991). «Developing the health care market». The Economic Journal, 101 (4): 1277-1286.

McMaster, R. (2001). «A veblenian-inspired critique of the «quasi-market» concept». International Journal of Social Economics, 28 (9):710-724.

- (2002). "The analysis of welfare state reform: Why the «quasi-markets» narrative is descriptively inadequate and misleading». Journal of Economic Issues, 36 (3): 769-794.

MÉnARD, C. (1995). «Markets as institutions versus organizations as markets? disentangling some fundamental concepts». Journal of Economic Behavior and Organization, 28 (2): 161-182.

- (1996). «On clusters, hybrids and other forms strange forms: The case of the french poultry industry». Journal of Institutional and Theoretical Economics, $152(1): 154-183$.

Musgrave, R. A. (1959). The theory of public finance. New York: McGraw-Hill.

- (1987). «Merit goods», en J. Eatwell, M. Milgate y P. Newman (eds.), The new palgrave: A dictionary of economics. Basingstoke: Palgrave Macmillan. OCDE (2005). Modernising government: The way forward. Paris: OECD.

PAstor, I. (2006). ¿La gestión privada en la administración pública? Madrid: Consejo Económico y Social.

Propper, C. (1993). «Quasi-markets, contracts and quality in health and social care: The U.S experience», en J. Le Grand y W. Barlett (eds.), Quasimarkets and social policy. London: MacMillan.

Siciliani, L. y Stanciole, A. (2013). «Bargaining and the provision of health services». The European Journal of Health Economics, 14 (3)ः 391-406.

Tambour, M. y Rehnberg, C. (1997). Internal markets and performance in Swedish health care. EFI.

Williamson, O. E. (1975). Markets and hierarchies: Analysis and antitrust implications. A study in the economics of internal organization. New York: The Free Press.

- (1985). The economic institutions of capitalism. New York: Free PressMacMillan. 